\title{
Cost Effectiveness of Sucroferric Oxyhydroxide Compared with Sevelamer Carbonate in the Treatment of Hyperphosphataemia in Patients Receiving Dialysis, from the Perspective of the National Health Service in Scotland
}

\author{
Florian S. Gutzwiller ${ }^{1} \cdot$ Alena M. Pfeil ${ }^{1} \cdot$ Zanfina Ademi $^{1} \cdot$ Patricia R. Blank $^{1} \cdot$ \\ Peter G. Braunhofer ${ }^{2}$ - Thomas D. Szucs ${ }^{1} \cdot$ Matthias Schwenkglenks $^{1}$
}

Published online: 3 September 2015

(c) The Author(s) 2015. This article is published with open access at Springerlink.com

\begin{abstract}
Background Hyperphosphataemia is common and harmful in patients receiving dialysis. Treatment options include noncalcium-based phosphate binders such as sevelamer carbonate (SC) and sucroferric oxyhydroxide (PA21).

Objective The aim of this study was to determine the health economic impact of PA21-based strategies compared with SC-based strategies, from the perspective of the Scottish National Health Service (NHS).

Methods A Markov model was constructed based on data from a randomised clinical trial comparing PA21 and SC. Model input parameters were derived from published literature, national statistics and unpublished sources. Costs (price year 2012) and effects were discounted at $3.5 \%$. Analysis with a lifelong time horizon yielded the incremental cost-effectiveness ratio (ICER), expressed as cost or savings per quality-adjusted life-year (QALY) gained or forgone. Deterministic and probabilistic sensitivity analysis was performed to explore uncertainties around assumptions and model input parameters.

Results In the base-case analysis, phosphorus reductions for PA21 and SC were 1.93 and $1.95 \mathrm{mg} / \mathrm{dL}$. Average undiscounted survival was estimated to be 7.61 years per patient in both strategies. PA21 patients accrued less QALYs (2.826) than SC patients (2.835), partially due to differential occurrence of side effects. Total costs were $£ 13,119$ and $£ 14,728$ for PA21 and SC, respectively (difference per patient of £1609). By using PA21 versus SC,
\end{abstract}

Florian S. Gutzwiller

fgutzwiller@gmx.ch

1 Institute of Pharmaceutical Medicine (ECPM), University of Basel, Klingelbergstrasse 61, 4056 Basel, Switzerland

2 Vifor Pharma AG, Glattbrugg, Switzerland one would save £174,999 (or £123,463 when including dialysis and transplantation costs) for one QALY forgone. A scenario modelling the nonsignificant reduction in mortality (relative risk 0.714 ) observed in the trial yielded an ICER for PA21 of $£ 22,621$ per QALY gained. In probabilistic sensitivity analysis of the base-case, PA21 was dominant in $11 \%$, and at least cost-effective in $53 \%$, of iterations, using a threshold of $£ 20,000$ per QALY gained. Conclusions The use of PA21 versus SC in hyperphosphataemic patients being intolerant of calcium-based phosphate binders may be cost saving and yields only very limited disadvantages in terms of quality-adjusted survival. PA21 appears to be cost-effective from the perspective of the Scottish NHS.

\section{Key Points for Decision Makers}

Control of hyperphosphataemia in end-stage renal disease (ESRD) patients receiving maintenance dialysis is clinically important. Sucroferric oxyhydroxide (PA21) is a new calcium-free polynuclear iron(III)-oxyhydroxide phosphate binder with a high phosphate binding capacity.

In patients receiving maintenance dialysis intolerant of calcium-based phosphate binders, compared with sevelamer carbonate treatment PA21 appears to be cost saving and appears to yield only very limited disadvantages in terms of quality-adjusted survival from the perspective of the Scottish National Health Service.

Due to lack of data, certain assumptions had to be made. Future studies are needed to provide evidence of long-term outcomes. 


\section{Introduction}

Hyperphosphataemia is a commonly encountered problem in patients with chronic kidney disease (CKD), particularly in its advanced stages, which occurs because of the inability of the dysfunctioning kidney to eliminate dietary phosphorus [1]. Additionally, there is an increased release of phosphorus from the bone due to dysregulation in calcium balance and hyperparathyroidism.

Hyperphosphataemia has been associated with a variety of long-term complications and can affect any organ system [2]. Prolonged hyperphosphataemia leads to soft-tissue and vascular calcification. Calcification of coronary arteries, cardiac valves, and pulmonary tissues is associated with cardiac disease, the leading cause of death in patients with kidney disease [1]. Therefore, it is important to prevent and treat hyperphosphataemia and maintain serum phosphorus levels within the recommended ranges.

The mainstay of treatment is dietary phosphorus restriction [1]; however, many patients require pharmacologic management with phosphate-binding drugs. Currently available phosphate-binding agents include aluminium hydroxide, magnesium carbonate, calcium carbonate (CC), calcium acetate, sevelamer carbonate (SC), and lanthanum carbonate (LC).

The British Renal Association guidelines recommend that in patients with kidney disease, serum phosphorus, if elevated, should be lowered towards the normal range, between 1.1 and $1.8 \mathrm{mmol} / \mathrm{L}(3.41-5.57 \mathrm{mg} / \mathrm{dL})$ [3].

In Scotland, first-line treatment of hyperphosphataemia consists primarily of CC. Patients intolerant of or not responsive to $\mathrm{CC}$ can be treated with a second-line therapy based on noncalcium-based phosphate binders. SC is a noncalcium-based phosphate binder and is currently the market leader in this class [4]. Sucroferric oxyhydroxide (PA21) is a new calcium-free polynuclear iron(III)-oxyhydroxide phosphate binder with a high phosphate binding capacity [5].

The authors aimed to determine the health economic impact of PA21 and SC as competing treatment options in end-stage renal disease (ESRD) patients receiving maintenance dialysis and with hyperphosphataemia. PA21 and SC were compared from the perspective of the Scottish National Health Service (NHS).

\section{Methods}

A Markov cohort model was constructed using TreeAge Pro 2014 Suite ${ }^{\circledR}$ (TreeAge Software, Inc., Williamstown, MA, USA) to assess the cost effectiveness of therapy with PA21 compared with SC. Given the potentially long-term consequences of the initial treatment decision, a lifelong time horizon was used.

\subsection{Efficacy and Safety Data}

Clinical model inputs were predominantly based on the efficacy and safety results of the PA-CL-05A/B trial. This trial studied 1041 (PA21 $n=694$, SC $n=347$ ) and 658 (PA21 $n=391, \mathrm{SC} n=267$ ) maintenance dialysis patients enrolled in the US as well as several European countries, including Austria, Belgium, Croatia, Czech Republic, Germany, Latvia, Lithuania, Poland, Romania and the UK. Patients had a history of hyperphosphataemia and of receiving stable doses of phosphate binders. In the PA-CL05A study, subjects were randomised in a 2:1 ratio to either PA21 or SC, respectively [6]. In both trial arms, study drug was titrated for safety and efficacy during a 12-week titration period, and patients then received maintenance treatment for an additional 12 weeks (stage 1, total duration 24 weeks). Subjects who completed stage 1 of the PA-CL-05A study at week 24 and who consented to participate in stage 2 $(n=100)$ were randomised to receive either PA21 or SC at the dose administered at the end of week 24, or low-dose PA21 for 3 weeks. In the PA-CL-05B trial [7], subjects continued to receive either PA21 or SC as allocated at randomisation into PA-CL-05A [6], at the same dose they were taking at the end of PA-CL-05A (week 24 for subjects who only participated in stage 1 and week 27 for subjects who participated in stage 2). Treatment with PA21 and SC continued for a further 28 weeks [7]. The primary endpoint was change in serum phosphorus level. The trial publications by Floege et al. reported the results of each phase of the trial separately [6,7]. Additionally, the second publication reported efficacy for the entire trial period (phases A and B combined), but focused on completers only [7]. In contrast, all PA-CL-05A/B-based parameters used in this analysis were based on the full analysis set $(n=1041)$, as available from a currently unpublished clinical study report. ${ }^{1}$ In this set, the PA-CL-05A/B trial (phases A and B combined) showed overall mean reductions of serum phosphorus of $1.93 \mathrm{mg} / \mathrm{dL}$ (95\% confidence interval [CI] 1.78-2.08) and $1.95 \mathrm{mg} / \mathrm{dL}$ (95\% CI 1.73-2.17) in the PA21 and SC arms, respectively (see Footnote 1 ). These reductions were taken into account in the base-case analysis. A nonsignificant survival difference (relative risk [RR] 0.714 for PA21 vs. SC; $95 \%$ CI 0.37-1.40) observed between the trial arms was taken into account in a scenario analysis (see Footnote 1).

\footnotetext{
1 Vifor Pharma. Clinical study report. Open-label, randomised, active-controlled, parallel group, multicentre phase 3 studies to investigate the long-term safety, tolerability and efficacy of PA21 compared with sevelamer carbonate in dialysis patients with hyperphosphataemia. An integrated report for study PA-CL-05A and extension study PA-CL-05B. May 2013 (personal communication).
} 


\subsection{Structure of the Markov Model}

The structure of the Markov model is shown in Fig. 1. The mortality effect due to elevated serum phosphorus, and the treatment effect of PA21 and SC on serum phosphorus, were the two principal mechanisms in the model. Patients started on either PA21 or SC treatment at a mean serum phosphorus starting level of $7.59 \mathrm{mg} / \mathrm{dL}$ (see Footnote 1). Initial survival was adapted to account for the increased risk of mortality associated with a high baseline serum phosphorus level [8]. The subsequent treatment-induced risk reduction was based on the mean reductions of phosphorus levels observed in the PA21 and SC arms of the PACL-05A/B trial (see Footnote 1), as reported above. The risk reduction was assumed to apply immediately after the initiation of treatment. Patients either continued their initial treatment or switched to the alternative treatment in case of withdrawal or sustained hyperphosphataemia. These options are represented by the six mutually exclusive health states of the model: on-target (patients continue and achieve target serum phosphorus levels), off-target (patients remain hyperphosphataemic), alternative treatment on-target (patients switched to alternative treatment achieve target phosphorus level), alternative treatment offtarget (patients switched to alternative treatment receive maximum drug dose but do not achieve target phosphorus levels), renal transplant (implying the end of hyperphosphataemia treatment), and death, with stage transitions at the end of each cycle. Markov cycle length was set at 1 month and half-cycle correction was implemented as appropriate. According to the United States Renal Data System (USRDS) report, the expected remaining lifespan is approximately 8 years for dialysis patients aged 40-44 years and approximately 4.5 years for those aged 60-64 years [9]. Mean age at model entry was 56 years. The model adopted a lifetime time horizon represented by a maximum of 44 years (528 cycles), corresponding to age 100 years. Costs were assessed from the third-party payer perspective of the Scottish NHS, i.e. indirect costs were not included. Costs and effects occurring after year 1 were discounted at $3.5 \%$. The primary endpoints of this analysis were undiscounted survival, quality-adjusted life-years (QALYs), and cost per QALY gained reported as incremental cost-effectiveness ratio (ICER).

\subsection{Base-Case Assumptions}

The modelled patient cohort was assumed to be intolerant to calcium-based phosphate binders. Due to high intolerance regarding calcium-based phosphate binders, we assumed that patients did not switch from a calcium-free to a calcium-based phosphate binder [10]. For the base-case analysis, the following additional assumptions were made.
1. The risk of mortality for treated patients was driven by a mean reduction of serum phosphorus of $1.93 \mathrm{mg} / \mathrm{dL}$ ( $0.62 \mathrm{mmol} / \mathrm{L}$, conversion factor 0.323 ) for PA21, and $1.95 \mathrm{mg} / \mathrm{dL}(0.63 \mathrm{mmol} / \mathrm{L}$, conversion factor 0.323$)$ for SC (see Footnote 1). RRs of mortality corresponding to these reductions of serum phosphorus levels were assigned and the resulting changes in mortality risk were applied to all patients receiving treatment, as calculation of the underlying average reductions also included patients who were 'off-target', i.e. experienced sustained hyperphosphataemia.

2. It was assumed that after the start of hyperphosphataemia treatment, patients were immediately 'ontarget' or 'off-target'.

3. The treatment-specific percentage of withdrawals due to treatment-emergent adverse events (TEAEs) seen in the PA-CL-05A/B trial was applied to patients 'ontarget' (distributed over 1 year, corresponding to the trial observation period). 'On-target' patients who withdrew from PA21 or SC due to TEAEs were assumed to switch to the other drug and were either 'on-target' (with treatment continuing at standard dose) or 'off-target' (with treatment continuing at maximum dose), based on the distribution of treatment success versus sustained hyperphosphataemia observed in the trial for each substance.

4. Patients with persistent hyperphosphataemia ('offtarget') switched to the other drug during half a year. After the switch from PA21 to SC, or vice versa, patients were also either 'on-target' (with treatment continuing at the standard dose) or 'off-target' (with treatment continuing at the maximum dose), according to the proportions seen in the entire trial (see Footnote 1). The same treatment-specific mean reductions of serum phosphorus as observed in the trial (see Footnote 1) were assumed $(1.93 \mathrm{mg} / \mathrm{dL}$ for PA21 and $1.95 \mathrm{mg} / \mathrm{dL}$ for SC).

5. The occurrence of transplantations was set to zero in the base-case analysis, and dialysis and transplantation costs were excluded because these costs were not significantly affected by the choice of phosphate binder. This decision has a precedent in National Institute for Health and Care Excellence (NICE) clinical guideline 157 [11]. The impact of dialysis and transplantation costs was addressed in a scenario analysis.

\subsection{Model Input Parameters}

An overview of all model input parameters, distinguishing base-case and scenario input parameters, is provided in Table 1. 

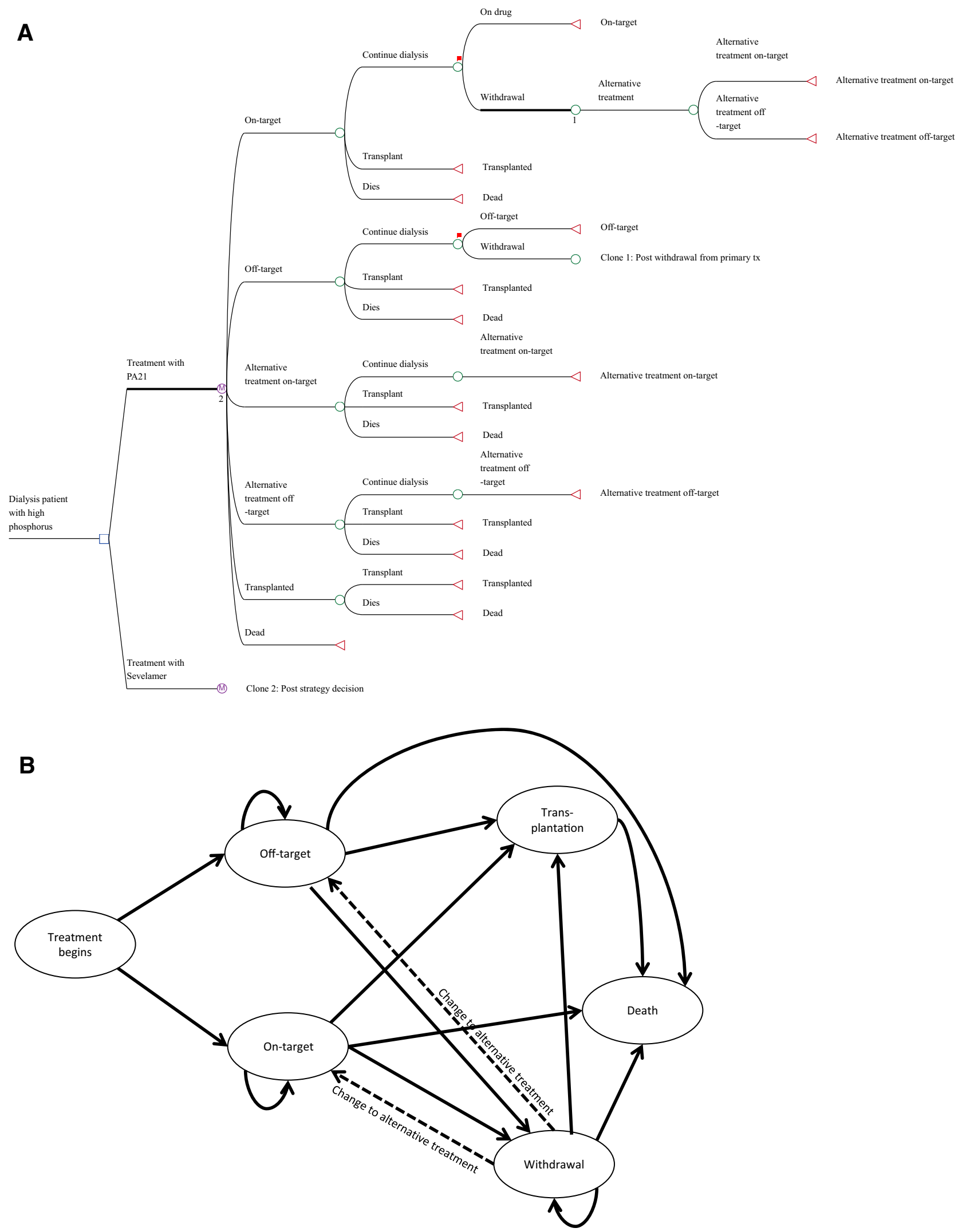

Fig. 1 Decision analysis model. a Structure of the Markov model; $\mathbf{b}$ generic structure of the hyperphosphataemia treatment pathway model with six mutually exclusive health states. PA21 sucroferric oxyhydroxide 


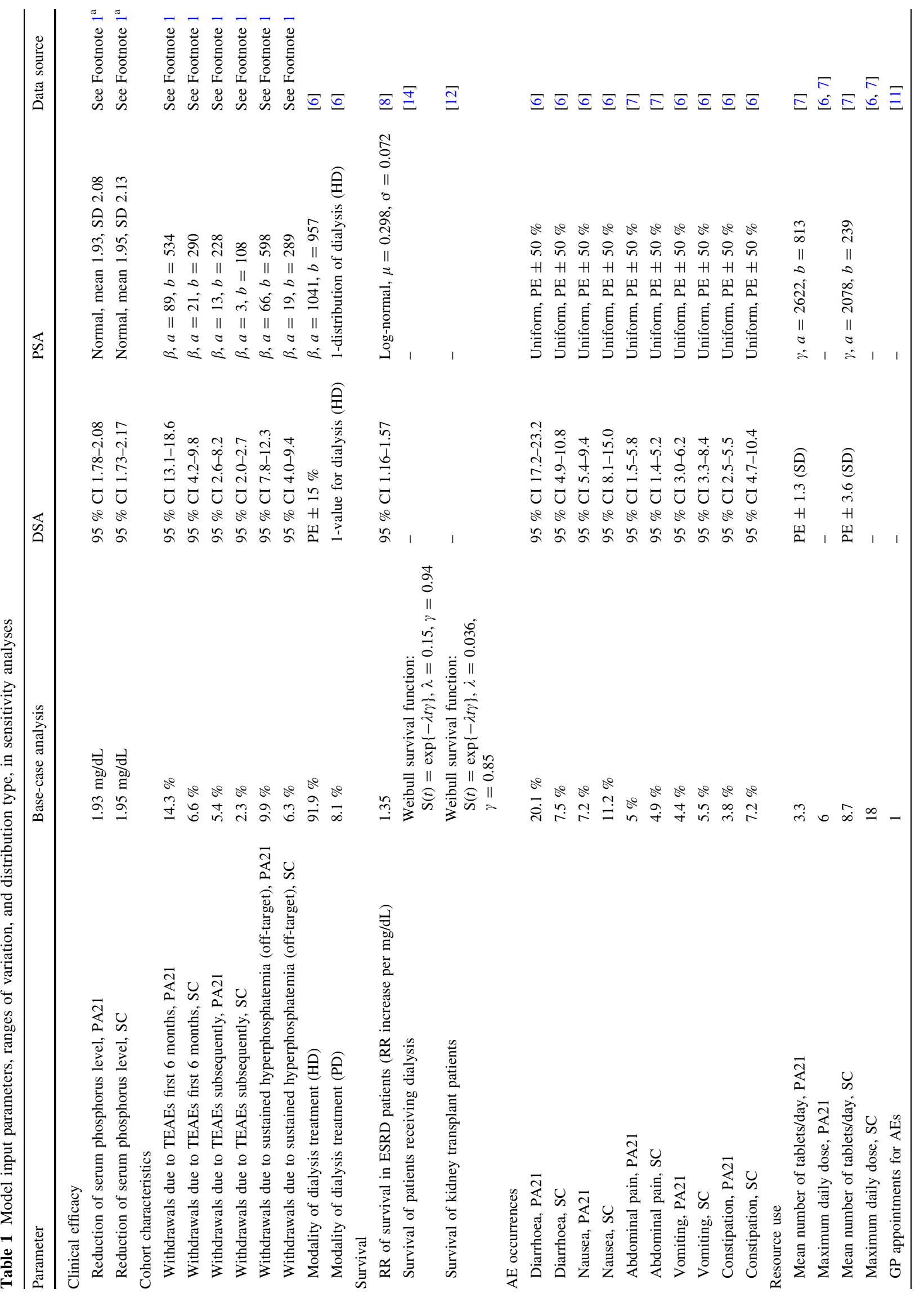




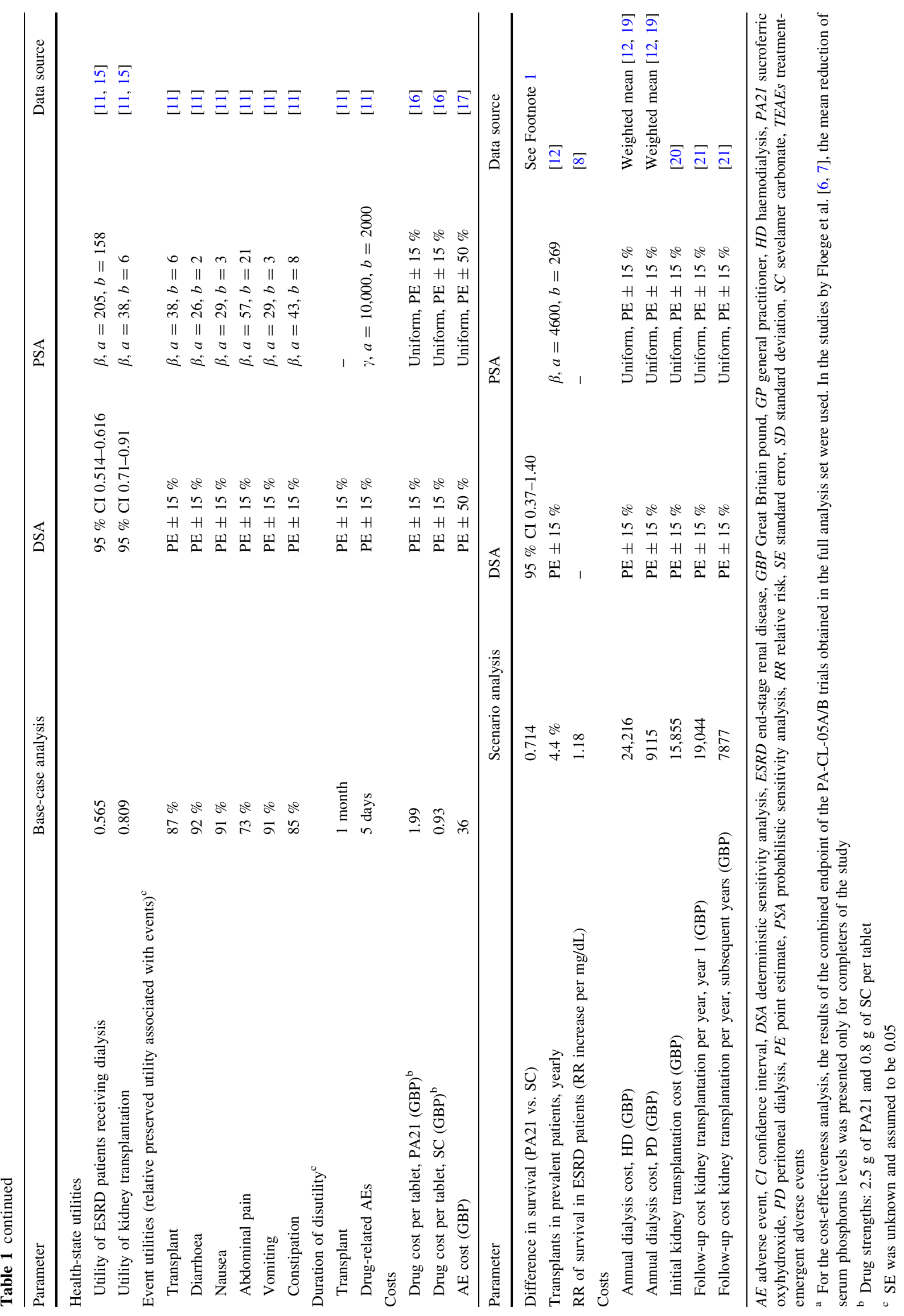


Fig. 2 a Survival curves reflecting survival for patients with ESRD, patients receiving dialysis and patients receiving a kidney transplant that has been extrapolated from the SRR report [12] and the UK RRR [14]. b Survival curves implemented in the model. $E S R D$ end-stage renal disease, $S R R$ Scottish Renal Registry, $U K$ RRR UK Annual Renal Registry Report, $P A 21$ sucroferric oxyhydroxide, $S C$ sevelamer carbonate
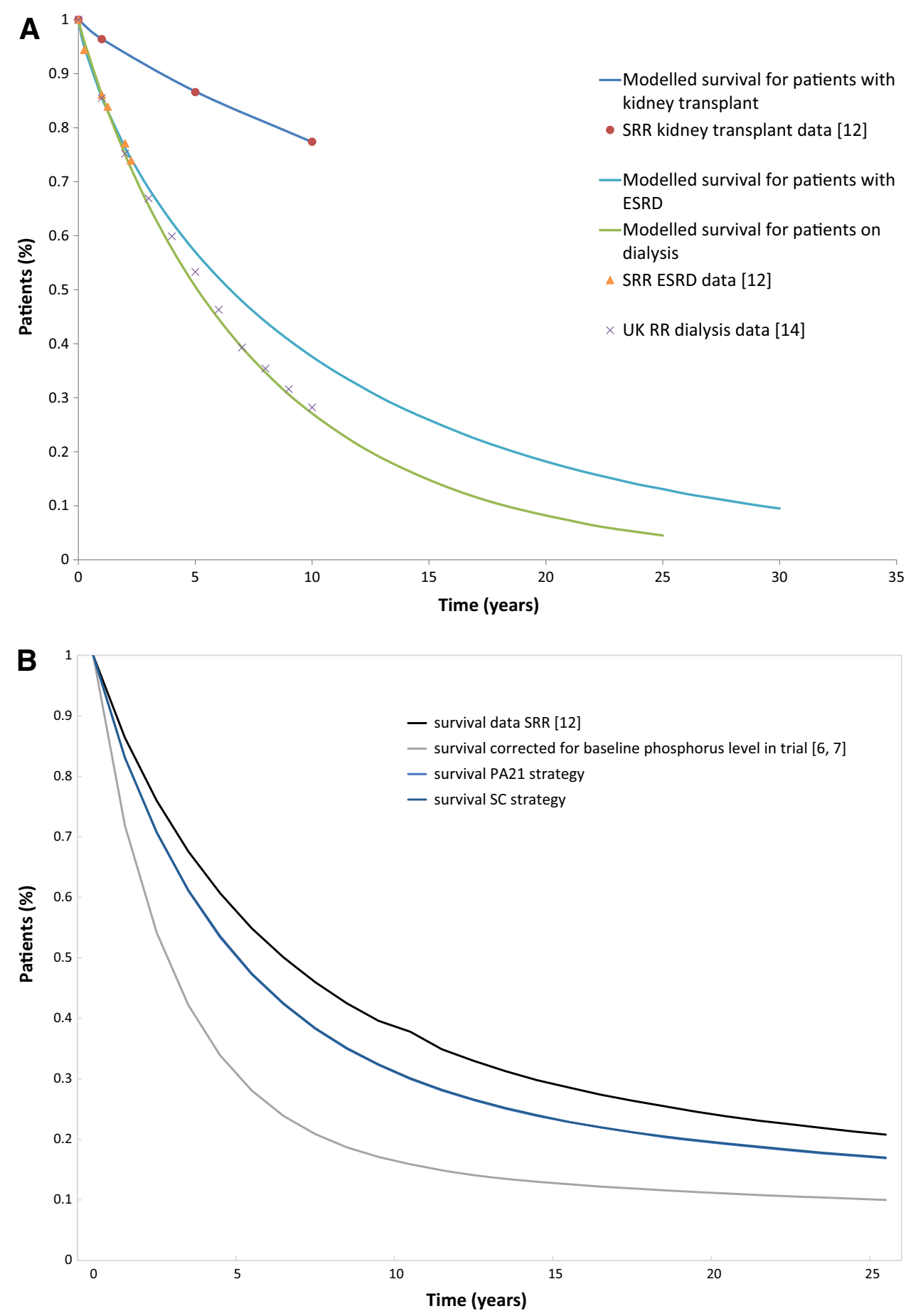

In order to provide a basis for modelling the impact of PA21 and SC treatment on survival, the following steps were undertaken (Fig. 2a). First, a Weibull distribution was defined to reflect the survival curve for patients with a kidney transplant, as reported in the Scottish Renal Registry (SRR) report (Table D3.1 in the report) [12]. Subsequently, a Weibull distribution [13] reflecting the survival of patients receiving dialysis, as available from the UK Annual Renal Registry report (Table 8.10 in the report)
[14], was implemented such that the joint survival curve of patients receiving dialysis and patients with a kidney transplant matched the overall survival estimates for all patients receiving renal replacement therapy, as available from the SRR report (Table C1.3 in the report) [12]. Importantly, the impact of serum phosphorus levels on mortality was set to zero before these steps were undertaken. Because the baseline phosphorus levels in the PACL-05A/B trial full-analysis population (average across 
arms, $7.59 \mathrm{mg} / \mathrm{dL}$ or $2.45 \mathrm{mmol} / \mathrm{L}$; conversion factor 0.323 (see Footnote 1)) were higher than in the SRR population $(4.86 \mathrm{mg} / \mathrm{dL}$ [12]), the 'starting' mortality of the modelled cohort was subsequently adjusted 'upwards' to reflect this difference. It was assumed that for every $1 \mathrm{mg} / \mathrm{dL}$ increase in serum phosphorus, the risk of mortality would increase by $35 \%$ (RR 1.35 ; $95 \%$ CI 1.16-1.57), based on a metaanalysis by Palmer et al. including studies that adequately controlled for confounding [8]. On this basis, and assuming the same RR per $1 \mathrm{mg} / \mathrm{dL}$ change, the phosphorus level changes observed in the trial could be applied (Palmer et al. also reported a second, more conservative estimate of the increase in mortality risk per $1 \mathrm{mg} / \mathrm{dL}$ increase in serum phosphorus of 1.18 (95 \% CI 1.12-1.25), partially based on less adequately controlled studies. This value was used in a scenario analysis [8]. The resulting survivor curves are shown in Fig. $2 b$.

Utility parameters were derived from published literature, and the utilities assumed for ESRD on dialysis and kidney transplantation were 0.57 and 0.81 , respectively. These values were random effects-based pooled weighted means of EQ-5D questionnaire-based, patient-elicited utilities from different countries, identified in a systematic review [15] and reanalysed for NICE guideline 157 [11]. Disutilities for the first month after transplantation and associated with TEAEs were taken from NICE guideline 157 [11].

Medical resource utilisation estimates of drug use and average pill burden, maximum daily doses and the use of dialysis (haemodialysis: $91.9 \%[N=957]$; peritoneal dialysis: $8.1 \%[N=84])$ were based on the PA-CL-05A/B trial (Table 1) [6]. Those patients who switched to the alternative treatment and were 'off-target' were assumed to receive the maximum daily dose. Patients who were 'ontarget' were assumed to receive the mean number of tablets per day (Table 1). The occurrence of TEAEs of phosphate binder treatment was derived from the PA-CL-05A/B trial data $[6,7]$. Only AEs with a frequency of at least $2 \%$ were included in the analyses because the occurrence of infrequent AEs could be driven by chance. Probability of transplantation was derived from the SRR [12].

The costs used in the base-case model are limited to the costs of both drug therapy and adverse events. British National Formulary data were used to extract the price for PA21 and SC [16]. TEAEs were assumed to incur one general practitioner (GP) appointment each [11] (costs reported by Curtis [17]). Laboratory and monitoring costs were the same for PA21 and SC and did not impact the ICER; therefore, these costs were not included in the model. Estimates of annual costs of dialysis were derived from the SRR report and the National Schedule of Reference Costs $[12,18]$. Transplantation costs were estimated according to the information available from the NHS and from two publications [19, 20, 21]. Costs (reported in pounds sterling [£]) were inflated to 2012 prices as necessary, using the 2012 Pay and Prices Index for hospital and community health services as estimated by the Personal Social Services Research Unit (PSSRU) [17].

\subsection{Sensitivity Analysis and Scenario Analyses}

To assess the influence of parameter uncertainty on model results, a series of deterministic (DSA) and probabilistic sensitivity analyses (PSA) were performed. All parameters underlying uncertainty were varied, with the exception of the number of GP appointments for adverse events (which were assumed to be one visit per TEAE, in line with NICE guideline 157 [11]). Variables were varied by their $95 \%$ $\mathrm{CI}$ or, if unavailable, by $\pm 15 \%$, as previously in an analysis comparable to ours [19] (Table 1). PSA (secondorder Monte Carlo simulation) was based on corresponding distributions (Table 1). Each sensitivity analysis was based on 10,000 sets of randomly drawn input parameters.

A first scenario analysis replaced the relative mortality risk estimate of 1.35 per $1 \mathrm{mg} / \mathrm{dL}$ increase in serum phosphorus level with a more conservative estimate of 1.18 [8]. In a second scenario analysis, dialysis and transplantation costs were included in the base-case analysis, while in a third scenario analysis mortality was modelled differently for the PA21 and SC strategies, based on the nonsignificant RR of mortality $(0.714$ for PA21 vs. SC; $95 \%$ CI 0.37-1.40) observed between the PA-CL-05A/B trial arms (see Footnote 1). Taking into account the trial observation period, this RR was applied for 1 year. The occurrence of transplantations, as well as dialysis and transplantation costs, were also included in this scenario. A fourth scenario analysis assessed the impact of increasing the costs of diarrhoea (£1500 instead of £36), an adverse event that was more common in the PA21 strategy. A fifth scenario analysis with an extreme assumption was added to gain an understanding of the potential impact of our assumption that patients would switch from PA21 to SC, or from SC to PA21, due to TEAEs or sustained hyperphosphataemia. In this analysis, patients were assumed (counterfactually, we expect) to remain untreated after withdrawal from their initial treatment.

\section{Results}

\subsection{Base-Case Analysis}

The average survival was estimated to be 7.61 years (undiscounted) per patient in both strategies. During the 
Table 2 Costs and quality-adjusted life-years obtained for the base-case and scenario analyses

\begin{tabular}{|c|c|c|c|c|c|c|c|c|c|c|c|c|}
\hline \multirow[t]{2}{*}{ Parameter } & \multicolumn{3}{|c|}{ Base-case (GBP) } & \multicolumn{3}{|c|}{$\begin{array}{l}\text { Base-case including dialysis } \\
\text { and transplantation costs } \\
\text { (GBP) }\end{array}$} & \multicolumn{3}{|c|}{$\begin{array}{l}\text { Scenario analysis of } \\
\text { nonsignificant relative risk } \\
(0.714) \text { of mortality (GBP) }\end{array}$} & \multicolumn{3}{|c|}{$\begin{array}{l}\text { Scenario analysis of } \\
\text { adverse event costs (GBP) }\end{array}$} \\
\hline & PA21 & $\mathrm{SC}$ & $\Delta$ & PA21 & $\mathrm{SC}$ & $\Delta$ & PA21 & $\mathrm{SC}$ & $\Delta$ & PA21 & $\mathrm{SC}$ & $\Delta$ \\
\hline Drug costs & 12,969 & 14,590 & -1621 & 9845 & 11,086 & -1241 & 6818 & 7194 & -376 & 12,969 & 14,590 & -1621 \\
\hline Adverse event costs & 150 & 138 & 12 & 114 & 105 & 9 & 79 & 68 & 11 & 2842 & 1561 & 1282 \\
\hline Transplantation costs & - & - & - & 34,414 & 34,497 & -83 & 24,296 & 22,720 & 1576 & - & - & - \\
\hline Dialysis costs & - & - & - & 87,552 & 87,773 & -220 & 60,956 & 56,926 & 4030 & - & - & - \\
\hline Total costs & 13,119 & 14,728 & -1609 & 131,925 & 133,461 & -1536 & 92,149 & 86,908 & 5241 & 15,811 & 16,149 & -338 \\
\hline QALYs & 2.826 & 2.835 & -0.009 & 5.045 & 5.057 & -0.012 & 3.545 & 3.313 & 0.232 & 2.826 & 2.835 & -0.009 \\
\hline
\end{tabular}

Costs are rounded values

GBP Great Britain pound, PA21 sucroferric oxyhydroxide, SC sevelamer carbonate, $Q A L Y S$ quality-adjusted life-years

analysis period of 44 years, patients in the PA21 strategy gained 2.826 QALYs, i.e. 0.009 QALYs less than in the SC strategy (2.835 QALYs) (Table 2). This small difference was partially due to the impact of TEAEs in the PA-CL05A/B trial (Table 1). Differences in the frequency of occurrence of TEAEs between the trial arms were significant for diarrhoea, nausea and constipation, and nonsignificant for abdominal pain and vomiting. Total costs were $£ 13,119$ and $£ 14,728$ in the PA21 and SC strategies, respectively (cost difference per patient of £1609). By using PA21 versus SC, one would save $£ 174,999$ for one QALY forgone.

\subsection{Sensitivity Analysis}

DSA was carried out to characterise the robustness of the base-case results, given parameter uncertainty (Fig. 3). Varying the mean number of tablets per day in the SC strategy had the highest impact on the ICER. The second most influential parameter was the number of tablets per day in the PA21 strategy, followed by drug cost per tablet (both strategies) and change in serum phosphorus level (both strategies). None of the remaining parameters, taken by themselves, had a significant impact on the ICER.

The PSA results showed mean costs of $£ 14,428$ for PA21 and £16,250 for SC [range PA21 £755-41,989; SC $£ 683-50,706]$. Mean QALYs were 3.111 for PA21 and 3.126 for SC [range PA21 0.155-8.991; SC 0.147-9.599]. The mean effect difference was 0.015 QALYs. In $11 \%$ of iterations, PA21 dominated SC, and in $53 \%$ of iterations it was at least cost-effective, using a threshold of $£ 20,000$ per QALY gained. A corresponding cost-effectiveness scatterplot and acceptability curve are shown in Fig. 4a. The scatterplot confirms the finding of a cost advantage for PA21, but no clear advantage for either treatment in terms of clinical effectiveness; it indicates a substantial degree of uncertainty.

\subsection{Scenario Analyses}

In the first scenario analysis, the lower relative mortality risk estimate of 1.18 per $1 \mathrm{mg} / \mathrm{dL}$ increase in serum phosphorus level reduced the cost difference between PA21 (£10,384) and SC (£11,658) to 11274 , and the difference in effectiveness to 0.005 QALYs (PA21 2.239, SC 2.244). The PA21 strategy would save £297,940 for one QALY forgone over a lifelong time horizon.

In the second scenario analysis, the base-case analysis was repeated but the occurrence of transplantations was now modelled and dialysis and transplantation costs were included. As previously, PA21 was less expensive than SC (£131,925 vs. $£ 133,461$; difference of $£ 1536$ ), but less QALYs were gained with PA21 than with SC (5.045 QALYs vs. 5.057 QALYs; difference of 0.012 QALYs). By using the PA21 strategy, one would save $£ 123,463$ for one QALY forgone over a lifelong time horizon.

In the third scenario analysis, the difference in effectiveness between the PA21 and SC strategies was based on the nonsignificant RR of mortality (RR 0.714 for PA21 vs. $\mathrm{SC}$ ) observed between the PA-CL-05A/B trial arms. In this scenario, the PA21 strategy was more expensive and accrued more QALYs than the SC strategy. Total costs amounted to £92,149 and £86,908 in the PA21 and SC strategies, respectively, resulting in a cost difference of $£ 5241$ over a lifelong time horizon. The gain in QALYs was 3.545 versus 3.313 (difference of 0.232 ) in favour of PA21. In this scenario, the ICER for PA21 compared with SC was $£ 22,621$. In $3 \%$ of scenarios, PA21 dominated SC, and in $27 \%$ of scenarios it was cost-effective (Fig. 5).

In the fourth scenario analysis, diarrhoea costs were increased to $£ 1500$. The cost difference is lower than in the base-case analysis ( $\$ 15,811$ vs. $£ 14,149$; difference of $£ 338$ ) and the difference in QALYs is the same (0.009) as in the base-case analysis (2.826 QALYs for PA21 vs. 2.835 QALYs for SC). By using the PA21 strategy, one would 
Fig. 3 Tornado diagram of deterministic sensitivity analysis addressing the impact of parameter uncertainty. $G B P$ Great Britain pound, ICER incremental cost-effectiveness ratio, $P A 21$ sucroferric oxyhydroxide, $Q A L Y$ qualityadjusted life-year, $S C$ sevelamer carbonate

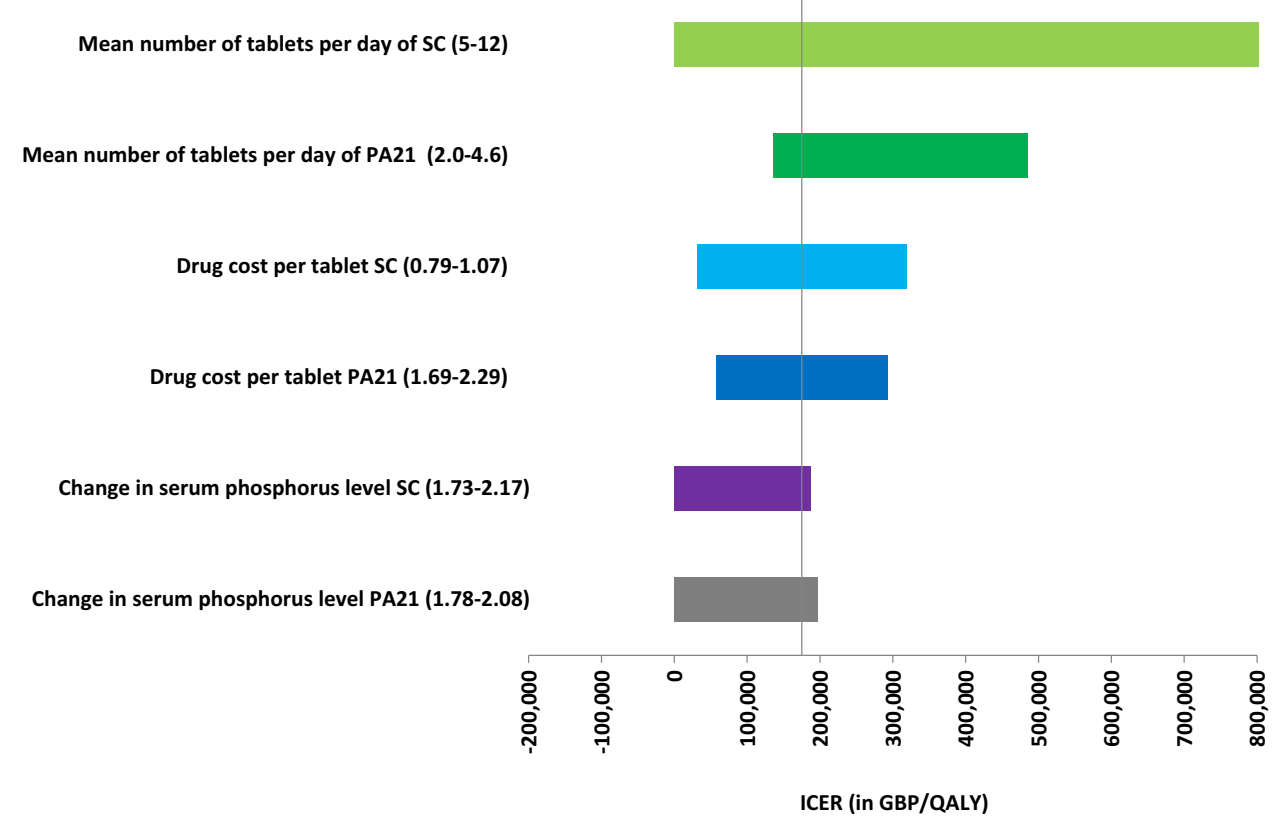

save less (£170,030) for one QALY forgone over a lifelong time horizon compared with the base-case (£36,810 vs. $£ 174,999)$.

In the scenario analysis with the extreme assumption that patients with TEAEs or sustained hyperphosphataemia would remain untreated after withdrawal from their initial treatment, the total costs for PA21 were lower than the total costs for $\mathrm{SC}$, and the difference in costs was higher than in the base-case (£9058 vs. £12,929; difference of £3871). The gain in QALYs was lower for both strategies compared with the base-case, but slightly more QALYs were gained with the SC strategy compared with the PA21 strategy (2.679 QALYs for SC and 2.533 QALYs for PA21). By using the PA21 strategy, one would save less for one QALY forgone over a lifelong time horizon compared wtih the base-case (£26,515 vs. £174,999).

\section{Discussion}

In ESRD patients receiving maintenance dialysis, over a lifelong time horizon, a PA21-based treatment strategy appears to incur lower costs than SC-based treatment, from the perspective of the Scottish NHS. QALYs accrued in the base-case model were marginally lower for the PA21 strategy. By using the PA21 strategy, one would save $£ 174,999$ (or £123,463 when including dialysis and transplantation costs) for one QALY forgone. The underlying small QALY loss is partially driven by differences in TEAE occurrence between treatment strategies. When modelling the nonsignificant reduction in mortality (RR 0.714 for PA21 vs. SC) observed in the PA-CL-05A/B trial in a scenario analysis, the PA21 strategy accrued more QALYs and was more expensive than the SC strategy, leading to an ICER of $£ 22,621$. The relative increase in costs on the PA21 side was due to improved survival and hence longer times on hyperphosphataemia and renal replacement therapy.

Additional sensitivity and scenario analyses generally confirmed these observations but indicated a substantial degree of uncertainty due to sensitivity to some model parameters. The DSA showed that pill burden, while being a serious problem for patient adherence [22, 23], also substantially impacted the ICER over a patient's lifetime. Also influential were the drug costs of PA21 and SC, as well as the change in mean serum phosphorus level (Fig. 3). Importantly, the model was not sensitive to changes in the number of withdrawals, health state utilities, utility decrements, proportion of patients with sustained hyperphosphataemia, or occurrence of TEAEs. Assuming a lower impact of serum phosphorus level change on mortality did not alter the direction of the results. Including the occurrence of transplantations, as well as costs for dialysis and transplantation, resulted in higher total costs, but an unchanged cost difference and similar QALYs accrued for both treatment strategies. The same trend was seen when a $500 \%$ increase in AE costs was assumed (Table 2).

Calcium-based binders have been the standard of care in phosphate-binding therapy for many years [24]. In cases where this first-line therapy is not indicated (e.g. in 
Fig. 4 Cost-effectiveness scatterplot and acceptability curve (base-case analysis). a Cost-effectiveness scatterplot of 10,000 bootstrap iterations for incremental cost and incremental effectiveness. Every dot represents one iteration of the simulation, and $95 \%$ of iterations are depicted in the circle. Parameters were simultaneously and randomly sampled from the probabilities, cost, and outcome distributions for each strategy to account for uncertainty in the base-case parameter estimates (probabilistic sensitivity analysis). b Cost-effectiveness acceptability curve presenting the probability that the strategy with sucroferric oxyhydroxide is cost-effective compared with the strategy with sevelamer carbonate for different willingness-to-pay values. $G B P$ Great Britain pound, $P A 21$ sucroferric oxyhydroxide, $Q A L Y s$ quality-adjusted lifeyears, WTP willingness-to-pay
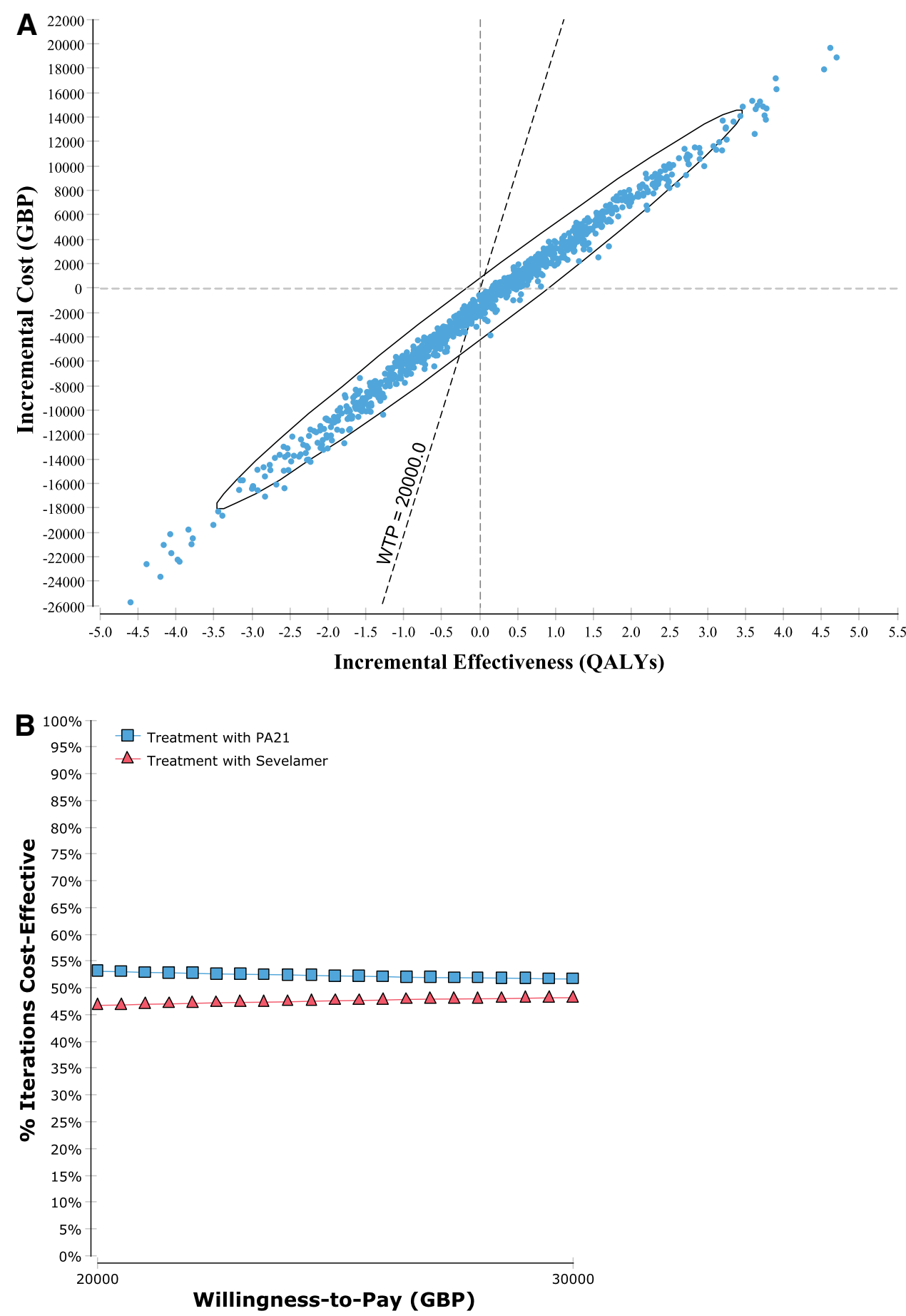

hypercalcaemic patients), noncalcium phosphate binders are recommended for use [24]. In terms of clinical effectiveness, there is evidence that $\mathrm{SC}$ is able to achieve similar reductions in serum phosphorus levels as $\mathrm{CC}$, with a similar pill burden $[25,26]$. Some reports have addressed the cost effectiveness of other noncalcium phosphate binders [27-32] but the current analysis is the first to assess the cost effectiveness of the novel drug PA21 in the treatment of hyperphosphataemia. The majority of data inputs could also be applied to the UK and therefore we believe that there would not be a big difference between our results and the results of a cost-effectiveness analysis performed from the perspective of the NHS in the UK.

Future research, ideally based on long-term observation, should focus on a possible association of reduced pill burden with improved compliance and adherence and resulting clinical effects, an aspect that could not be considered in this analysis due to a lack of data, but which has 
Fig. 5 Cost-effectiveness scatterplot (scenario analysis) of 10,000 bootstrap iterations for incremental cost and incremental effectiveness. Every $d o t$ represents one iteration of the simulation. $95 \%$ of iterations are depicted in the circle. Parameters were simultaneously and randomly sampled from the probabilities, cost and outcome distributions for each strategy to account for uncertainty in the base-case parameter estimates (probabilistic sensitivity analysis). $G B P$ Great Britain pound, $Q A L Y s$ quality-adjusted life-years, WTP willingness-topay

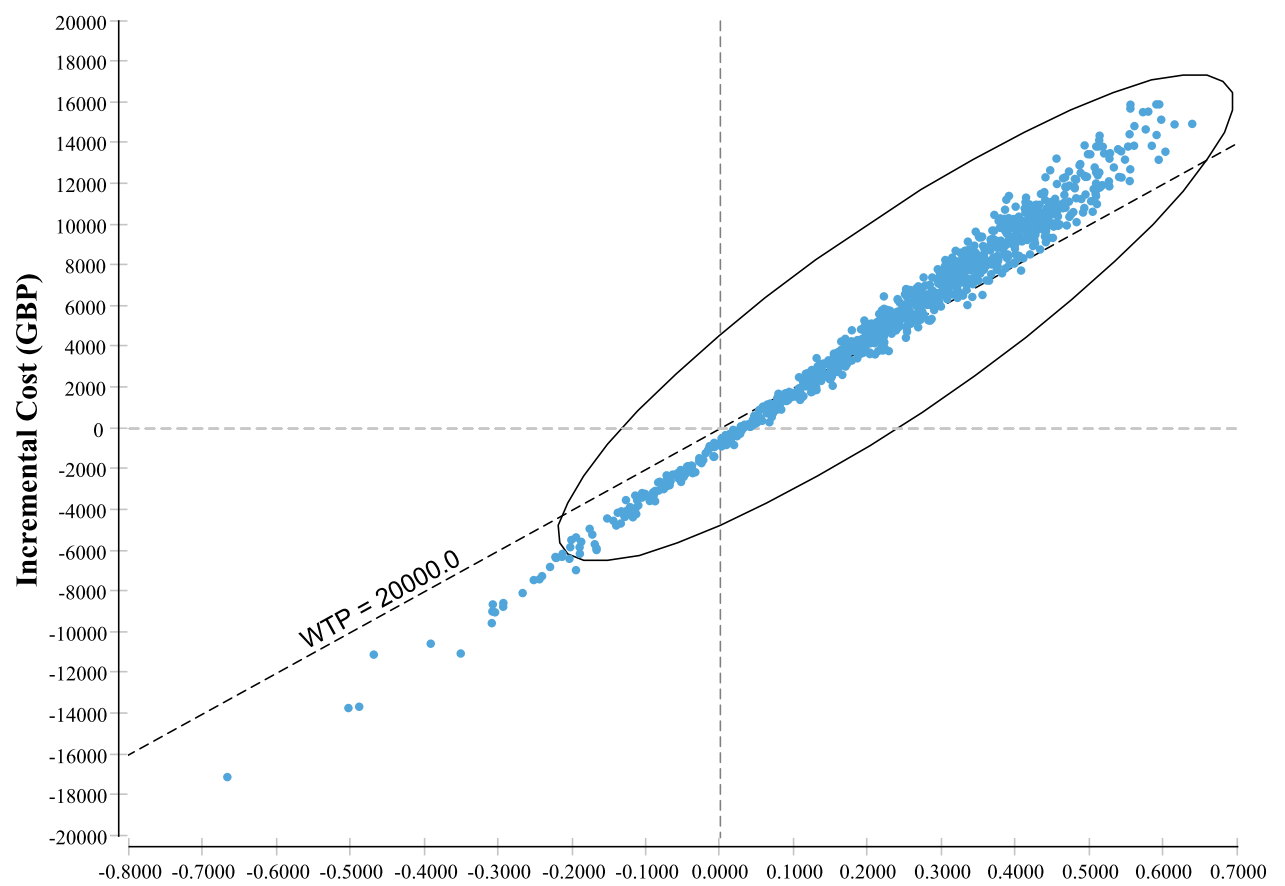

Incremental Effectiveness (QALYs) been addressed elsewhere [33]. The existence of differences in adherence between different phosphate binders and associated effects on clinical outcomes should be assessed in a real-life setting.

Our study has a number of limitations. The data sources available to us were limited in scope. Some assumptions had to be made where no data were available. In the study by Floege et al. [7], the mean reduction of serum phosphorus level was presented for completers of the PA-CL05A/B trial. Therefore, efficacy data for the cost-effectiveness analysis were obtained from the unpublished clinical study report (see Footnote 1), which covers the full analysis set. In this analysis set, the mean reduction of serum phosphorus levels was slightly higher for SC than for PA21. This renders our cost-effectiveness analysis more conservative than if the values for completers reported in Floege et al. [7] had been used (the mean reduction of serum phosphorus levels in completers was higher for PA21 than for SC). The proportions of patients 'on-target' versus 'off-target', as well as the withdrawal rates observed in the PA-CL-05A/B trial $[6,7]$, on which our results are based, may differ from those to be expected under real-world conditions. We did not include other phosphate binders in our cost-effectiveness analysis because SC was the only phosphate binder being compared with PA21 in a head-to-head trial. In current clinical practice, combination therapy with phosphate binders is common [34, 35]; however, published data on how different phosphate binders should exactly be combined and what the clinical implications would be is rather vague
[11]. A strategy combining PA21 and SC in the same patient and at the same time could not be modelled without introducing an uncontrollable amount of uncertainty. Therefore, we decided to only model strategies representing monotherapy, with a possibility of switching to the alternative treatment in the case of nonresponse or withdrawal due to TEAEs. After switching, the same treatmentspecific efficacy as observed in the trial (reduction in serum phosphorus levels, $1.93 \mathrm{mg} / \mathrm{dL}$ for PA21 and $1.95 \mathrm{mg} / \mathrm{dL}$ for SC) was assumed due to a lack of published data. In the case of continued nonresponse, administration of the maximum recommended dose was assumed. The assumed patient pathway may not fully reflect routine practice. Under the extreme assumption that patients would remain untreated after withdrawal from their initial treatment, the savings for one QALY forgone by using the PA21 strategy were reduced to $£ 26,515$ in comparison with $£ 174,999$ in the base-case analysis. PA21 was disfavoured here due to the higher withdrawal rates than for SC observed in the PA-CL-05A/B trial. However, given the long-term complications of hyperphosphataemia, we expect the assumption of no further treatment after withdrawal from PA21 or $\mathrm{SC}$ to be counterfactual for most patients. Cardiovascular calcification negatively impacts on cardiovascular risk and contributes to mortality in ESRD patients [36]. SC has been shown to slow down the progression of cardiovascular calcification compared with calcium-based phosphate binders [37]. As PA21 is also a calcium-free phosphate binder and has shown a nonsignificant survival advantage compared with SC (RR of mortality 0.714 ; $95 \%$ CI $0.37-1.40$ ), 
we assumed PA21 to be noninferior to SC with respect to positive effects on cardiovascular calcification, cardiovascular risk and survival not directly driven by the reduction in serum phosphorus. This was a prerequisite of building our base-case analysis on the change in serum phosphorus levels observed in the trial. Our base-case analysis did not include costs of dialysis and transplantation. However, these costs were close to equivalent in the two treatment strategies, as shown in a scenario analysis. This might also be the case for other costs caused by conditions that are not influenced by the reduction in serum phosphorus level. Because comorbidities and concomitant medications were not reported in the clinical trial publications $[6,7]$, it was difficult to assess the comparability of patients in the clinical trial and patients in Scotland. However, in the unpublished study report, it was reported that the proportion of patients with cardiac disorders was similar in both treatment arms and no significant differences regarding concomitant medications were reported. Due to a lack of data regarding cardiovascular event risks, we could not assess the number of avoided cardiovascular events based on the reduction in serum phosphorus level. However, because the difference in the mean reduction of serum phosphorus levels was low, we assumed that the effect of PA21 versus SC on the occurrence of cardiovascular events would be limited.

\section{Conclusions}

The use of PA21 in patients with hyperphosphataemia and intolerant to calcium-based phosphate binders may be cost saving when compared with SC, and yields only very limited disadvantages in terms of quality-adjusted survival. PA21 appears to be cost-effective from the perspective of the Scottish NHS.

\section{Compliance with Ethical Standards}

Funding This study was supported by a research grant from Vifor Pharma Ltd, Switzerland.

Conflicts of interest Florian S. Gutzwiller, Matthias Schwenkglenks, Thomas D. Szucs, Zanfina Ademi, Patricia R. Blank and Alena M. Pfeil have received research grants from Vifor Pharma Ltd, Switzerland. Peter G. Braunhofer is an employee of Vifor Pharma Ltd, Switzerland.

Author contributions Florian S. Gutzwiller and Alena M. Pfeil contributed to the conception and design of the study, data acquisition, analysis and interpretation of data, drafting the article and final approval. Matthias Schwenkglenks contributed to the conception and design of the study, analysis and interpretation of data, revising the article and final approval. Thomas D. Szucs, Zanfina Ademi and Patricia R. Blank contributed to the conception and design of the study, interpretation of data, revising the article and final approval.
Peter G. Braunhofer contributed to the conception and design of the study, revising the article and final approval.

Open Access This article is distributed under the terms of the Creative Commons Attribution-NonCommercial 4.0 International License (http://creativecommons.org/licenses/by-nc/4.0/), which permits any noncommercial use, distribution, and reproduction in any medium, provided you give appropriate credit to the original author(s) and the source, provide a link to the Creative Commons license, and indicate if changes were made.

\section{References}

1. Tonelli M, Pannu N, Manns B. Oral phosphate binders in patients with kidney failure. N Engl J Med. 2010;362(14):1312-24.

2. Block GA, Hulbert-Shearon TE, Levin NW, Port FK. Association of serum phosphorus and calcium $\times$ phosphate product with mortality risk in chronic hemodialysis patients: a national study. Am J Kidney Dis. 1998;31(4):607-17.

3. Mactier R, Hoenich N, Breen C. The Renal Associationhaemodialysis guidelines. Petersfield: The Renal Association; 2009.

4. European and US Renvela Sales Data. http://www.imshealth.com and http://www.drugs.com/stats/renvela. Accessed 15 Apr 2015.

5. Hergesell O, Ritz E. Stabilized polynuclear iron hydroxide is an efficient oral phosphate binder in uraemic patients. Nephrol Dial Transplant. 1999;14(4):863-7.

6. Floege J, Covic AC, Ketteler M, Rastogi A, Chong EM, Gaillard S, et al. A phase III study of the efficacy and safety of a novel iron-based phosphate binder in dialysis patients. Kidney Int. 2014;86(3):638-47.

7. Floege J, Covic AC, Ketteler M, Mann JFE, Rastogi A, Spinowitz $B$, et al. Long-term effects of iron-based phosphate binder, sucroferric oxyhydroxide, in dialysis patients. Nephrol Dial Transplant. 2015;30(6):1037-46.

8. Palmer SC, Hayen A, Macaskill P, Pellegrini F, Craig JC, Elder GJ, et al. Serum levels of phosphorus, parathyroid hormone, and calcium and risks of death and cardiovascular disease in individuals with chronic kidney disease: a systematic review and meta-analysis. JAMA. 2011;305(11):1119-27.

9. Collins AJ, Foley RN, Herzog C, Chavers BM, Gilbertson D, Ishani A, et al. Excerpts from the US Renal Data System 2009 Annual Data Report. Am J Kidney Dis. 2010;55(1 Suppl 1):S1-420 (A6-7).

10. Wang Y, Xie G, Huang Y, Zhang H, Yang B, Mao Z. Calcium acetate or calcium carbonate for hyperphosphataemia of hemodialysis patients: a meta-analysis. PLoS One. 2015;10(3):e012137.

11. National Institute for Clinical Excellence. Hyperphosphataemia in chronic kidney disease. Manchester: National Institute for Clinical Excellence; 2013.

12. Scottish Renal Registry report 2013. Edinburgh: NHS National Services Scotland; 2014.

13. Carroll KJ. On the use and utility of the Weibull model in the analysis of survival data. Control Clin Trials. 2003;24(6):682-701.

14. The Renal Association UK Renal Registry Report 2013. Bristol: National Health Service England; 2013.

15. Liem YS, Bosch JL, Hunink MG. Preference-based quality of life of patients on renal replacement therapy: a systematic review and meta-analysis. Value Health. 2008;11(4):733-41.

16. Joint Formulary Committee. British National Formulary 69. British National Formulary; 2015. 
17. Curtis L. Unit costs of health and social care 2012. Kent: Personal Social Services Research Unit, University of Kent; 2012. Report No.: 978-1-902671-82-6.

18. NHS reference costs: financial year 2011 to 2012. Department of Health; 2012

19. National Health Service. Cost-effectiveness of transplantation. NHS Blood and Transplant. 2009. http://www.organdonation.nhs. uk/newsroom/fact_sheets/cost_effectiveness_of_transplantation. asp. Accessed 2 Nov 2013.

20. Hamidi V, Andersen MH, Oyen O, Mathisen L, Fosse E, Kristiansen IS. Cost effectiveness of open versus laparoscopic livingdonor nephrectomy. Transplantation. 2009;87(6):831-8.

21. Kerr M, Bray B, Medcalf J, O'Donoghue DJ, Matthews B. Estimating the financial cost of chronic kidney disease to the NHS in England. Nephrol Dial Transplant. 2012;27(3):iii73-80.

22. Chiu YW, Teitelbaum I, Misra M, de Leon EM, Adzize T, Mehrotra R. Pill burden, adherence, hyperphosphatemia, and quality of life in maintenance dialysis patients. Clin J Am Soc Nephrol. 2009;4(6):1089-96.

23. Wang S, Alfieri T, Ramakrishnan K, Braunhofer P, Newsome BA. Serum phosphorus levels and pill burden are inversely associated with adherence in patients on hemodialysis. Nephrol Dial Transplant. 2014;29(11):2092-9.

24. Sprague SM. A comparative review of the efficacy and safety of established phosphate binders: calcium, sevelamer, and lanthanum carbonate. Curr Med Res Opin. 2007;23(12):3167-75.

25. Braun J, Asmus HG, Holzer H, Brunkhorst R, Krause R, Schulz $\mathrm{W}$, et al. Long-term comparison of a calcium-free phosphate binder and calcium carbonate: phosphorus metabolism and cardiovascular calcification. Clin Nephrol. 2004;62(2):104-15.

26. Shaheen FA, Akeel NM, Badawi LS, Souqiyyeh MZ. Efficacy and safety of sevelamer. Comparison with calcium carbonate in the treatment of hyperphosphatemia in hemodialysis patients. Saudi Med J. 2004;25(6):785-91.

27. Park H, Rascati KL, Keith MS, Hodgkins P, Smyth M, Goldsmith $\mathrm{D}$, et al. Cost-effectiveness of lanthanum carbonate versus sevelamer hydrochloride for the treatment of hyperphosphatemia in patients with end-stage renal disease: a US payer perspective. Value Health. 2011;14(8):1002-9.

28. Bernard L, Mendelssohn D, Dunn E, Hutchison C, Grima DT. A modeled economic evaluation of sevelamer for treatment of hyperphosphatemia associated with chronic kidney disease among patients on dialysis in the United Kingdom. J Med Econ. 2013;16(1):1-9.

29. Plagemann T, Prenzler A, Mittendorf T. Considerations about the effectiveness and cost effectiveness of therapies in the treatment of hyperphosphataemia. Health Econ Rev. 2011;1(1):1.

30. St Peter WL, Fan Q, Weinhandl E, Liu J. Economic evaluation of sevelamer versus calcium-based phosphate binders in hemodialysis patients: a secondary analysis using centers for Medicare and Medicaid services data. Clin J Am Soc Nephrol. 2009;4(12):1954-61.

31. Vegter S, Tolley K, Keith MS, Lok CE, Soroka SD, Morton AR. Cost-effectiveness of lanthanum carbonate in the treatment of hyperphosphatemia in dialysis patients: a Canadian payer perspective. Clin Ther. 2012;34(7):1531-43.

32. Brennan A, Akehurst R, Davis S, Sakai H, Abbott V. The costeffectiveness of lanthanum carbonate in the treatment of hyperphosphatemia in patients with end-stage renal disease. Value Health. 2007;10(1):32-41.

33. Ramakrishnan K, Braunhofer P, Newsome B, Lubeck D, Wang S, Deuson $\mathrm{J}$, et al. The economic impact of improving phosphate binder therapy adherence and attainment of guideline phosphorus goals in hemodialysis patients: a medicare cost-offset model. Adv Ther. 2014;31:1272-86.

34. Mehrotra R, Martin KJ, Fishbane S, Sprague SM, Zeig S, Anger $\mathrm{M}$, et al. Higher strength lanthanum carbonate provides serum phosphorus control with a low tablet burden and is preferred by patients and physicians: a multicenter study. Clin J Am Soc Nephrol. 2008;3(5):1437-45.

35. Kramann R, Floege J, Ketteler M, Marx N, Brandenburg VM. Medical options to fight mortality in end-stage renal disease: a review of the literature. Nephrol Dial Transplant. 2012;27(12):4298-307.

36. Rastogi A. Sevelamer revisited: pleiotropic effects on endothelial and cardiovascular risk factors in chronic kidney disease and endstage renal disease. Ther Adv Cardiovasc Dis. 2013;7(6):322-42.

37. Biggar P, Kettler M. Sevelamer carbonate for the treatment of hyperphosphatemia in patients with kidney failure (CKD III-V). Expert Opin Pharmacother. 2010;11(16):2739-50. 